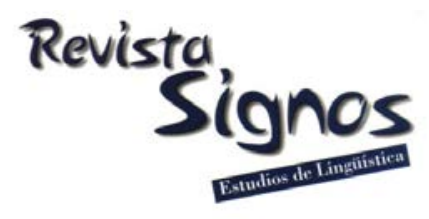

\title{
Las escrituras profesionales: Dispositivos argumentativos y estrategias retóricas
}

\author{
Professional writings: Argumentative devices and rhetorical \\ strategies
}

\author{
Elvira Narvaja de \\ Arnoux \\ UNIVERSIDAD DE BUENOS AIRES \\ ARGENTINA \\ elviraarnoux@gmail.com
}

\author{
Mariana di Stefano \\ UNIVERSIDAD DE BUENOS AIRES \\ ARGENTINA \\ marianadist@gmail.com
}

\author{
María Cecilia Pereira \\ UNIVERSIDAD DE BUENOS AIRES \\ ARGENTINA \\ mcpereira.pereira6@gmail.com
}

Recibido: 11/I/2016 / Aceptado: 04/VII/2016

\section{Resumen}

El presente trabajo busca destacar la importancia, para una pedagogía de la escritura en la formación profesional, de los 'dispositivos argumentativos' propios de los diferentes ámbitos profesionales. Se parte de considerar que las discursividades de estos diferentes ámbitos implican modos peculiares de conceptualizar los datos, de relacionar lo particular con lo general y de responder al problema específico que los interpela. Si bien esos modos de conceptualizar no son ajenos a las herramientas semióticas, en especial verbales e icónicas, pueden pensarse como previos a la puesta en discurso y que dan lugar a dispositivos argumentativos orientadores que se exponen, finalmente, en los géneros propios de dichas prácticas, con el despliegue de las estrategias retóricas correspondientes. En este trabajo se ilustra el funcionamiento de 'dispositivos argumentativos' a partir de ejemplos propios de cuatro prácticas profesionales que se caracterizan por ejercerse centralmente a través de la producción discursiva: son las asumidas por psicoanalistas, sacerdotes, periodistas y abogados. Se analizan también dispositivos normativos que regulan las discursividades profesionales, ya que las respectivas profesiones atienden a esas prescripciones y al hacerlo exponen la representación que tienen de su práctica.

Palabras Clave: Dispositivo argumentativo, prediscursivo, formación profesional, pedagogía de la escritura, géneros discursivos. 


\begin{abstract}
This paper seeks to highlight the importance for teaching of writing in the vocational training of their own 'argumentative devices' of different professional fields. It is considered that the discursivities of these areas involve different ways of conceptualizing data, to link the particular with the general and to answer specific problem that challenges them. While these ways of conceptualizing are no strangers to the semiotic tools, especially verbal and iconic, they can be thought as pre-discursive and that they give rise to argumentative guiding devices which are explained finally in their own genres of such practices, with the deployment of the appropriate rhetorical strategies. In this work, the 'argumentative devices' are illustrated as of examples from four professional practices, characterized by centrally exercised through discursive production: they are assumed by psychoanalysts, priests, lawyers and journalists. Regulatory provisions governing professional discursivities are also analyzed, because the respective professions pay attention to these requirements, and in doing so, expose the representation they have of their practice.
\end{abstract}

Key Words: Argumentative device, prediscursive, vocational training, writing teaching, discursive genres.

\title{
INTRODUCCIÓN
}

Las discursividades propias de los diferentes ámbitos profesionales implican modos peculiares de conceptualizar los datos, de relacionar lo particular con lo general, es decir con el universo de saberes y normas constituidos en ese marco, y de responder a lo que interpela al experto. Podemos pensar esas operaciones intelectuales como previas a la puesta en discurso pero que se articulan en dispositivos argumentativos que orientan y limitan la producción de los textos, aunque las estrategias retóricas varíen según los géneros. En este trabajo buscamos definir el concepto de dispositivo argumentativo, e ilustrar su funcionamiento a partir de ejemplos propios de cuatro prácticas profesionales con el objetivo de destacar su importancia para una pedagogía de la escritura en la formación profesional. En un mundo de mutaciones rápidas, en el que los géneros varían por exigencia de nuevas prácticas y soportes, consideramos importante abordar en la reflexión áulica no solo los funcionamientos genéricos sino también las operaciones cognitivas y los dispositivos dominantes en cada área, más estables, en los que aquellos se afirman ${ }^{1}$.

Las profesiones a las que hemos atendido en este trabajo se caracterizan por ejercerse centralmente a través de la producción discursiva: son las asumidas por psicoanalistas, sacerdotes, periodistas y abogados. En el caso de los psicoanalistas y abogados, la escritura está fundamentalmente destinada a sus pares. En sacerdotes y periodistas se piensa en un público más amplio y, en general, heterogéneo.

Partimos de considerar distintos tipos de actividades que realizan los profesionales: mientras algunas son respuestas a los desafíos cotidianos propios de su profesión (como dictar sentencia para un juez, o responder el abogado a una consultoría jurídica 
o presentar el periodista la noticia que va a publicar un diario, o exponer el psicoanalista un material clínico, o escribir el sacerdote una homilía destinada a una celebración litúrgica), otras actividades pueden surgir de requerimientos institucionales (como elaborar un código para el jurista o, en el caso del periodista, establecer una normativa para la presentación de notas en el medio en que actúa). Por otro lado, hay actividades que no son exclusivas de un campo profesional específico, pero pueden ser realizadas por los profesionales como un modo de acompañar en determinadas situaciones la práctica profesional (como participar en una conferencia de prensa o redactar como experto una nota periodística).

En este trabajo nos hemos centrado en el primer tipo de actividades que señalamos más arriba, es decir, dentro del 'conjunto de géneros' (Bazerman, 2009), las que constituyen respuestas a desafíos cotidianos propios de la profesión y que la definen. Pero al identificar el dispositivo argumentativo veremos en un ejemplo, el del discurso sacerdotal, cómo también incide en las producciones que incluiríamos en los otros grupos, en este caso en el tercero.

Debemos señalar que los procedimientos a los que se apela no son individuales, sino que resultan de lo que la profesión ha ido naturalizando por considerarlos más eficaces para abordar los problemas que habitualmente se presentan en relación con la función que los expertos de ese campo deben cumplir. Estos modos de conceptualizar, que definen los dispositivos argumentativos, es decir, los modos de entrelazar lo particular y convocar lo general para llegar a determinadas conclusiones, pueden encarnarse en variados géneros pero hay siempre uno o algunos que funcionan como modelos porque son centrales en la práctica y exhiben los dispositivos argumentativos dominantes (en los ejemplos que vamos a abordar: el relato clínico; la homilía; la noticia; y la sentencia). Esto quiere decir que algunos géneros tienen una función destacada porque ponen en juego las exigencias y los recursos de la profesión para intervenir satisfactoriamente en las prácticas. Incluso, pueden llegar a hacer aportes que incidan en el avance del conocimiento en ese campo o en ámbitos sociales más amplios.

Así, un relato clínico puede interrogar el saber psicoanalítico mostrando sus límites; una homilía puede proponer una actualización del mensaje que interpele a los fieles y a la institución generando cambios significativos; una noticia puede dar la información de tal manera que influya en la percepción que una comunidad tiene de determinados fenómenos sociales; y una sentencia puede sentar jurisprudencia. Pero el aspecto que busca resaltar este trabajo es que, más allá de las exigencias del género discursivo, a lo que se dedican numerosos estudios a partir de metodologías cuantitativas o cualitativas, es necesario reconocer las operaciones intelectuales a las que recurren los miembros de una determinada profesión para atender a las demandas del campo. 
A continuación, establecemos, en primer lugar, una relación entre la noción de 'dispositivo argumentativo' que estamos proponiendo y reflexiones actuales acerca de 'lo prediscursivo', que sirven para elaborar el marco teórico. En segundo lugar, nos referiremos a los textos normativos que regulan las discursividades profesionales y su anclaje en tradiciones específicas, ya que es importante atender a ellas cuando las respectivas profesiones lo hacen y al hacerlo exponen la representación que tienen de su práctica. Nos detendremos, finalmente, en los dispositivos argumentativos dominantes en cada profesión: el caso, la analogía, el razonamiento entimemático y la ilustración con hechos probados de la sujeción o transgresión a la norma.

Las observaciones que realizamos surgen de un contacto asiduo con los profesionales y con sus producciones (en situación de asesoramiento o de seminarios de formación) y del análisis de los manuales que regulan las respectivas discursividades (en el marco de los estudios que realizamos sobre las expresiones actuales de la tradición retórica). No provienen de pruebas realizadas con el objetivo de obtener datos ni del acopio exhaustivo y el análisis cuantitativo de los discursos producidos por los profesionales. Adoptamos metodológicamente un enfoque cualitativo e ilustramos a partir de textos seleccionados por su representatividad. Lo que proponemos es inferir ( $\mathrm{y}$, en gran medida, conjeturar) los modos de pensar de los profesionales $\mathrm{o}$, en términos generales, los modos de vincular los datos con los saberes y valoraciones del propio campo para poder suministrar una resolución adecuada a una situación problemática.

Queremos destacar la necesidad de pensar en la formación de profesionales innovadores, para lo cual las generalizaciones provenientes de corpus extensos y el carácter muchas veces prescriptivo que les asignamos en relación con los géneros, si bien facilitan la tarea redaccional, pueden funcionar como un obstáculo en la reflexión (creativa) sobre la respuesta al problema o la situación planteada.

\section{En torno a lo prediscursivo}

Cuando estudiamos el proceso de elaboración de una tesis (Arnoux, 2009) insistimos en la importancia de la progresiva construcción de una representación global del trabajo en la cual elementos constituyentes son -además de la dimensión valorativa, el eje temático y el destinatario- la orientación argumentativa y los ajustes entre investigación y textualización, ya que estas dos últimas actividades implican órdenes distintos. En los escritos profesionales también son necesarios los ajustes entre los modos de razonar, para proponer una respuesta a lo que la situación solicita, y el despliegue discursivo, que puede tener pautas convencionales fijas. A esa diferencia alude un texto normativo, cuando se señala en relación con el campo jurídico: 
"Pasaremos a considerar la forma de realizar una demanda que, en su preparación, no conviene que guarde la distribución definitiva que hemos dado en párrafos anteriores" (Falcón, 1997: 19).

Sin embargo, tanto en la preparación como en la textualización, los dos aspectos (modos de razonar y puesta en discurso) interactúan y convocan al hacerlo la memoria compartida de la comunidad profesional.

Por su parte, Bazerman (2015: 185) asigna importancia a las expectativas genéricas que determinan los modos legítimos, para una determinada actividad, de recolección y selección de los materiales y de los modos de representar los hechos en un espaciotiempo específicos y de razonar a partir de ellos: "El cronotopo u ontología de cada género y sus formas adecuadas de representación implican también una epistemología, una manera de conocer". Señala, entonces, la ontología y la epistemología asociadas a los géneros. Así una nota periodística parte de normas profesionales que "son, en esencia, directrices epistemológicas para la recolección, verificación y registro de informaciones" (Bazerman, 2015: 186). Y en las normas jurídicas de prueba reconocemos:

"epistemologías y prácticas que aceptan ciertos tipos de testimonios y documentos probatorios como legítimos y jurídicamente válidos, mientras excluyen otros" (Bazerman, 2015: 186).

Si bien es cierta la relación entre esas ontologías, epistemologías y los géneros, pensamos que los exceden ya que son modos profesionales de pensar que pueden aparecer en diversos géneros. En el campo jurídico, por ejemplo, lo que Bazerman señala puede reconocerse en sentencias pero también en alegatos, demandas, defensas o apelaciones. Paveau (2006) preferiría hablar, más que de expectativas genéricas, de 'prediscurso'. Si bien señala que este designa datos anteriores al discurso que son movilizados por él, al delimitarlo Paveau (2006: 14) lo caracteriza como:

"[...] un conjunto de marcos prediscursivos colectivos, que tienen un rol instruccional para la producción e interpretación del sentido en discurso. Entiendo por prediscurso contenidos semánticos (en el sentido amplio de cultural, ideológico, enciclopédico), es decir, saberes, creencias y prácticas y no solamente formas, ya que esto me conduciría del lado de los géneros del discurso tal como son formulados por Bajtín."2

Es evidente que como en toda actividad humana, en la profesional esos marcos intervienen. Dentro de ellos, la autora considera (Paveau, 2006: 187) los que llama "organizadores textuales-cognitivos", es decir, "formas cuyo funcionamiento se sitúa a medio camino entre la elaboración mental y la construcción textual. [...] tienen una dimensión cognitiva pues resultan de un formateo en el espíritu (shaping) y una dimensión textual porque se inscriben en ordenamientos textuales". Reconoce, como más solicitados en el corpus que ha analizado, la tipología, la metáfora y la antítesis. 
Nosotros preferimos hablar, a partir de los discursos profesionales, de dispositivos argumentativos, que implican determinada selección de los datos que se van a considerar y una relación con lo general (el saber del campo, la norma, la doctrina, la posición del medio), que va a llevar a que se oriente hacia determinado tipo de conclusión. Como señalamos los dispositivos argumentativos sostienen la discursividad, aunque esta pueda desplegarse diferentemente según los géneros.

Podemos considerar que en las profesiones que abordamos hay un dispositivo general usual que es la ilustración de lo general (dentro de la argumentación por lo particular, en términos de Perelman \& Olbrechts-Tyteca, 1970), que lleva a seleccionar los datos que se inscriben en él y a desechar los otros: tal hecho remite al complejo de Edipo, este otro muestra la misericordia divina, aquel puede ser caracterizado como robo porque transgrede la norma de respeto a la propiedad, y el otro muestra la incapacidad del gobierno de controlar la protesta social. Pero este dispositivo es básico y puede funcionar, particularmente, en el trabajo profesional rutinario. Sin embargo, lo que define al profesional es la capacidad de responder adecuadamente a un problema, es decir a algo que escapa a la mera ilustración porque presenta aspectos que cuestionan lo aceptado, porque plantea posibilidades alternativas, porque las causas no pueden definirse con claridad, porque hay un vacío legal a atender o porque lo que hay que elaborar como noticia resulta controversial.

En Psicoanálisis, el caso (Passeron \& Revel, 2005) es la forma de producir un saber nuevo a partir de relevar los desajustes de los datos respecto del saber de la clínica. En la predicación, la analogía (Mieville, 1983; Borel, 2000; Gardes-Tamine, 2011) que se construye entre los datos de la lectura establecida institucionalmente y la situación presente permite actualizar el mensaje religioso e interpretar desde el aquí y ahora la lectura bíblica. En el periodismo, la evaluación de la información va a construir una representación de la noticia que buscará imponerse a sus lectores. En derecho, la sucesión de hechos probados va mostrando o la transgresión o la sujeción a la(s) norma(s); si estas resultan insatisfactorias (son incompletas o entran en contradicción entre ellas) para juzgar lo acontecido nos encontramos también frente al caso.

\section{Dispositivos normativos y tradiciones de escritura}

En algunas de las profesiones que consideramos abundan los manuales o textos normativos de diverso alcance que regulan la producción discursiva: las que ejercen sacerdotes, periodistas y abogados. Estos textos son instrumentos lingüísticos (Auroux, 1994), elaborados en comunidades discursivas específicas, que inciden en las prácticas profesionales. Estas pueden apelar a instrumentos más generales -como gramáticas o diccionarios-, pero habitualmente, cuando la producción discursiva es importante en esa actividad, generan los instrumentos propios, que en algunos casos pueden también suministrar una síntesis de los otros (como en Belluscio, 2006). A veces aparecen como comunicaciones precarias destinadas al uso interno, por ejemplo, 
del aparato jurídico o de las redacciones de los diarios, pero en general tienden a una publicidad mayor. Están centrados en la selección de los datos a considerar en la elaboración del escrito y en los aspectos genéricos, particularmente composicionales y estilísticos.

En el ámbito jurídico, la regulación es fuerte sobre todo en lo referido a la estructura del texto, a los datos que no pueden ser obviados y a la manera de exponerlos. Sus fuentes son, en algunos casos, los códigos procesales y sus reglamentos, además del fuerte peso de la tradición retórica en esta comunidad. En los géneros jurídicos nucleares, los que se destacan son los mecanismos de construcción de un juicio fundado en el derecho vigente sobre un caso concreto.

Por su parte, los manuales referidos a las prácticas sacerdotales se inscriben en la larga duración. La serie de las artes de predicar, que es la designación que los engloba, se inicia en el siglo IV con De doctrina cristiana de San Agustín y atiende a variados aspectos: la preparación (análisis del texto base de las Sagradas Escrituras y relación con la situación coyuntural en que va a enunciarse el discurso), la redacción (eje interpretativo, planes textuales, estilo), los modos de memorizar y la actio, que considera las exigencias de los discursos orales.

En el caso de los periodistas, los manuales de estilo aparecen en la década del setenta del siglo pasado y tienen un importante desarrollo en los noventa (Arnoux, Blanco \& di Stefano, 1999); con el nuevo siglo se publican los manuales que consideran el soporte electrónico y se abren a prácticas no profesionales (Arnoux, 2015a). Así como los primeros se interesaban por los aspectos normativos gramaticales y ortográficos, los manuales de nuevo tipo los eluden y privilegian la diversidad de géneros que posibilita Internet y los requerimientos redaccionales de la pantalla. La noticia, como hipergénero que engloba a los géneros de información, ocupa un lugar central. En los psicoanalistas, si bien hay algunos textos que circulan en relación con la escritura (Gitaroff, 2010) es, a diferencia de los anteriores, fundamentalmente la práctica iniciada en la escritura freudiana la que regula esa producción.

Considerar estos manuales o las regularidades que podemos reconocer en las prácticas o los modelos a los que apelan nos permite apreciar cómo se perciben, desde dentro de la profesión, los requerimientos de los géneros propios. Los manuales o tratados tienden a generar un saber práctico en relación, particularmente, con los aspectos redaccionales: "Antes de confeccionar una demanda, uno debe conocer la estructura de la misma, esto es, cada una de las partes del escrito y el orden en que se suceden", afirma Falcón (1997: 15). También con los requerimientos institucionales:

"En la provincia de Buenos Aires, el sumario de la demanda ha sido ampliado (ampliación que se extiende a cualquier escrito inicial) requiriéndose otros datos..." (Falcón, 1997: 16). 
Pero no se detienen en los dispositivos argumentativos aunque podemos inferirlos por las normas que establecen para la producción de los textos; de allí el interés de consultarlos.

En la actualidad, la posibilidad de reunir amplias bases de datos monolingües y multilingües y recurrir a la tecnología informática para relevar las características comunes y las alternancias posibles ha llevado a establecer generalizaciones que prescriben los rasgos genéricos a distintos niveles conduciendo a una mayor fijación de los formatos. Esta preocupación se asocia con los requerimientos de la traducción automática y con el objetivo de la producción informática de textos o la automatización de la redacción (Roy, 2015). En el campo jurídico, incluso, se tienden a establecer:

"sistemas expertos basados en reglas que utilizan técnicas de inferencia lógica para crear modelos de razonamiento legal para la toma de decisiones automatizada" (Borja Albi, 2007: 148).

Estos procedimientos se proponen normalizar y normativizar las prácticas discursivas y en algunos casos aspiran a tener un alcance global. Lo que hay que reconocer es que, en muchos casos, los productos que estudian ya han sido regulados, como señalamos, por manuales específicos que tienen una respetable antigüedad.

\section{Dispositivos argumentativos}

Los modos de conceptualizar y razonar propios de cada práctica -que implican selección de los datos pertinentes, modos peculiares de puesta en relación con los saberes del campo (teóricos, doctrinarios, normativos, ideológicos) y orientación hacia una conclusión- dan lugar a dispositivos argumentativos específicos que inciden en la redacción. Los dispositivos sostienen lo central de los géneros profesionales (diferenciados según la esfera de actividad), que en algunos de ellos corresponde a una zona textual determinada. Así, en la presentación de materiales clínicos para una supervisión es el relato en forma de escenas dialogadas en las que se selecciona lo que funciona como síntoma de una perturbación y los esbozos de interpretación del analista (no la presentación del caso ni las razones por las que fue derivado el paciente). En la homilía no es la lectura previa ni el ruego final sino el vínculo interpretativo entre los datos que se seleccionan de la lectura y los que se seleccionan de la situación que vive la comunidad de fieles. En el discurso jurídico de la demanda o la sentencia no es el detenido y cuidado encabezamiento sino el juicio fundamentado en el derecho vigente sobre hechos que han de narrarse atendiendo a la pertinencia y a la orientación argumentativa global que se le dé al escrito. Y en el discurso periodístico, el relato del acontecimiento, y no los comentarios que puedan completarlo. 
Lo particular puede provenir del discurso de los pacientes, de los textos sagrados, de los clientes o de los cables; y puede ser visto como ejemplo de lo general (ilustración) o como lo que cuestiona lo general e impone nuevas hipótesis (caso), o como el foro (lo conocido) o el tema (lo nuevo) de la analogía, o como los datos que permiten llegar a determinada conclusión (acompañados de pruebas), o como los hechos cuyos rasgos significativos se seleccionan siguiendo una valoración global que tiene en cuenta la posición del medio.

Hablamos de 'dispositivos argumentativos' (Arnoux, 2012), porque la selección de lo particular y los modos de relación con lo general están argumentativamente orientados hacia una conclusión. La designación da cuenta, además, de que lo discursivo los considera, aunque los despliegues textuales varíen.

A continuación, vamos a analizar los dispositivos argumentativos en los discursos de cada una de las profesiones indicadas.

\subsection{El caso en Psicoanálisis}

En Psicoanálisis, muchos de los escritos que se producen y circulan ligados a la práctica profesional siguen la dinámica de la presentación de pacientes, que surge de la tradición médica (Burdiles, 2015) y encuentra su expresión más acabada en el relato clínico. Tanto en este como, por ejemplo, en los materiales clínicos para la supervisión, los historiales clínicos institucionales, o los artículos de revistas científicas podemos reconocer el dispositivo argumentativo 'caso' aunque las diferencias genéricas sean importantes. Consideremos, por ejemplo, las estrategias composicionales de dos de ellos:

En el relato clínico:

- Marco de la relación entre el terapeuta y el paciente

- Presentación del paciente

- Discurso narrativo que integra dos historias:

o La historia del paciente

o La historia de la terapia

- Conjeturas que se abren a posibles interpretaciones

En el material clínico destinado a la supervisión ${ }^{3}$ :

- Breve presentación del paciente, cómo llegó (posible derivación)

- Primera entrevista (transcripción de algún fragmento)

- Fragmento de otras sesiones (no hay cierre)

(Pueden encontrarse puntualizaciones acerca de las etapas de la terapia en las que alternan fragmentos comentativos y "transcripciones") 
Si bien los dos géneros son distintos -incluso las secuencias predominantes lo son, en un caso narración y en el otro, escenas dialogadas- el dispositivo argumentativo que concluye con un saber conjetural o apela a él es el del caso. Este tiene un primer movimiento intelectual, donde domina la ilustración, en el que un hecho se asocia con otros similares y se los considera (explícitamente o no) expresión, en el campo que estamos analizando, de una perturbación psíquica ya conocida. Hay un segundo movimiento que admite los desajustes entre los hechos y la teoría clínica a la que se recurrió, destacando lo que no responde a las verdades admitidas hasta ese momento. $\mathrm{Y}$ un tercer movimiento revisa los datos, interroga otros aspectos del saber psicoanalítico y propone una respuesta a lo planteado o apela a ella. Si estos movimientos no se dan, nos quedamos en el ejemplo o en la ilustración (Pereira, 2011) y no conformamos un caso (Jolles, 1972), que es lo realmente interesante para la comunidad discursiva, ya que hace avanzar la reflexión y permite reencauzar la terapia. De allí los rasgos que se señalan del caso en general: es singular (en su doble sentido de particular y excepcional), plantea problemas, reclama una solución, es decir, la instauración de un marco nuevo de razonamiento, es un enigma que debe ser resuelto (Passeron \& Revel, 2005). También lo conjetural de su resolución admite un nuevo recorrido interpretativo (reformulación discursiva del caso fuente) realizado por el mismo locutor u otro.

Garzoli (1996), terapeuta argentina, en uno de sus artículos publicado en una revista especializada en Psicoanálisis, presenta el caso 'July', una paciente alcohólica cuyo tratamiento llevó a la analista a interrogar distintas conceptualizaciones sobre la transferencia. Según relata Garzoli (1996: 327), July había sido “una 'paciente ejemplar' durante los dos primeros años" del tratamiento que, de acuerdo con los saberes propios del campo, le había propuesto (primer movimiento: el éxito ilustra lo ya sabido). No obstante, en el inicio de sus vacaciones que se empalmaban con las que, al mes siguiente, tomaría la terapeuta, la paciente comienza nuevamente a beber (segundo movimiento: muestra que el saber convocado ha sido insuficiente o inadecuado para resolver la situación de la paciente: "Los conceptos de neurosis de transferencia, de psicosis de transferencia y de perversión de transferencia - esta última unificada con la de los adictos- no se adecuaban por sus características a mis comprobaciones clínicas"). La analista estudia la situación y considera que la interrupción futura del tratamiento es lo que genera la caída otra vez en el alcoholismo. Interpreta entonces que si bien la paciente había pedido ayuda, cosa que en cambio no suelen hacer los adictos, el análisis realizado constituía para la paciente "una forma de continuar, desesperanzada y omnipotentemente, con sus ciclos de ‘desintoxicación' y recaídas". La analista conjetura que “ella se había convertido algo para manejar (como la bebida)" y que había estado realizando un "seudoanálisis", lo que impone cambios en la terapia: "Reencauzado entonces el proceso, pensando en las características observables del vínculo establecido, surgió en mí la necesidad de 
intentar conceptualizar su configuración, distinta de la que instala cualquier otro cuadro psicopatológico" (tercer movimiento: aporte teórico al conocimiento de los rasgos del alcohólico). Revisa, entonces, la noción de transferencia para el caso de los pacientes adictos y propone una serie de rasgos que la distinguen de la transferencia del perverso.

\subsection{La analogía en la predicación cristiana}

Si en el caso se articula lo general con lo particular y a partir de los desajustes se interroga lo general, en la actualización del mensaje propia de la predicación se va de lo particular (la lectura bíblica) a lo particular (la situación de la comunidad) gracias a un procedimiento analógico. Este se basa en la relación entre 'dos realidades heterogéneas' unidas por una 'propiedad global común' que el mismo discurso construye o que se asienta en saberes socialmente extendidos (Miéville, 1983).

Habitualmente se señala que en la analogía, el foro es más conocido, próximo y concreto que el tema, que es lo nuevo que hay que iluminar o cuya comprensión hay que facilitar. Sin embargo, en este caso, la lectura previa como las referencias a la situación presente entran en una dinámica en la que alternan y comparten su situación de foro y tema. El universo del texto sagrado es conocido porque acaba de ser leído, pero está más alejado de la experiencia de la audiencia; en cambio, los datos de la situación presente son más próximos, pero no la clave interpretativa doctrinaria que les asigna un sentido. Unos y otros se van iluminando mutuamente en el transcurso del discurso. La analogía permite la entrada interpretativa al texto primero a la vez que actualiza el mensaje presentando los datos del presente como semejantes a los de la 'lectura' (Arnoux, 2015b). Es este juego intelectual, del cual se deriva una posición doctrinal respecto de lo que vive la comunidad de fieles, lo que constituye el dispositivo argumentativo que al sacerdote le permite responder al desafío fundamental que sus prácticas discursivas le imponen más allá de los géneros.

Sin embargo, hay un género en el que este procedimiento cristaliza, la homilía, que parte de una lectura, que debe ser explicada tendiendo a mostrar cómo la Biblia nos habla aquí y ahora. Retóricamente tiene los rasgos de una reformulación explicativa. Y puede responder a diferentes esquemas textuales (Fernández, 2009: 123130):

1- motivación, desarrollo, conclusión; 2- afirmación, objeción, aclaración; 3- preguntas personales; 4- premisa evidente y preguntas abiertas (luego de un "pero" que muestre que no se han sacado las consecuencias esperadas); 5- pregunta, respuesta detallada, síntesis; 6- planteo, expectativa creciente, respuesta implícita; 7- desarrollo con hilo estructural (frase, versículo o estribillo); 8-enriquecimiento de significado palabra por palabra (de una frase); 9- explicación (exégesis sencilla) parte por parte. 
Por otra parte, el discurso debe desplegarse siguiendo un estilo que lo acerque al auditorio gracias, entre otros, a su dialogismo, a la presencia de un lenguaje coloquial propio de la cultura a la que pertenecen los que participan en la celebración, y al recurso a las imágenes (Francisco, 2013).

El modo de reflexión analógico permea muchas de las producciones discursivas que surgen de ese ámbito, aunque no correspondan al sistema de géneros propios de la comunidad religiosa. Para ilustrarlo nos referiremos a un escrito que apareció recientemente como nota periodística firmada por una autoridad de la comunidad.

En Argentina hubo una polémica porque el Papa Francisco obsequió un rosario bendecido a Milagros Sala, una luchadora social encarcelada inmediatamente después de la asunción de un nuevo gobierno en la provincia de Jujuy, en diciembre de 2015. Era cuestionada por manejos considerados poco claros respecto de fondos públicos que recibía de las autoridades anteriores. El gesto del Papa suscitó polémicas, tanto en los medios como en el clero. Monseñor Víctor Fernández utilizó en una nota periodística de opinión el habitual razonamiento analógico, que aplica primero a otros hechos de actualidad y, luego, a sucesos de la Biblia. Todos son expresiones del principio de la misericordia, que no se enuncia pero al que se alude y que legitima la argumentación:

"Es coherente que el Papa haya enviado un rosario a una presa que todavía no está en condiciones de recibir una condena, porque de hecho hizo lo mismo con culpables ya condenados por delitos muy graves. Es más: ha visitado cárceles y ha repartido rosarios en lugares repletos de criminales peligrosos. El mismo San Juan Pablo II visitó en la cárcel a un extremista islámico, sin que esto implicara justificar sus crímenes.

No debería llamar la atención que esto despertara resquemores ideológicos y políticos. Jesús comía y bebía con pecadores públicos, corruptos, gente despreciable, y por ello la gente supuestamente impecable decía con amarga ira: "Ahí tienen un comilón y un borracho, amigo de publicanos y pecadores" (Mt. 11.19). Y cuando le reprochaban un gesto misericordioso, Jesús preguntaba: “¿Por qué toman a mal que yo sea bueno?” (Mt. 20.15).” (La Nación, 19/2/2016)

Como vemos, en una nota periodística aparece un modo de razonar típico de la predicación, al que está habituado el autor. Las analogías utilizan la gradación tanto en relación con los personajes condenables (presa que todavía no está en condiciones de recibir una condena / condenados por delitos graves / criminales peligrosos /extremista islámico / pecadores públicos, corruptos, gente despreciable) como los admirables (el Papa Francisco / San Juan Pablo II / Jesús). Pero lo fundamental es la analogía entre el gesto del Papa y el gesto de Jesús. Y este se asienta en fragmentos de lecturas que le asignan al dispositivo el peso de lo doctrinario. 


\subsection{El razonamiento entimemático en la elaboración de la noticia periodística}

Los periodistas gráficos construyen la noticia, la elaboran discursivamente, a partir de cables, conferencias de prensa, de la observación de otros medios o de la búsqueda personal de información. Podemos decir que lo que define a la profesión es el tratamiento de la información y su conformación en noticia. Bonnet (2015) señala que Robert Park, que fundó la escuela de sociología de Chicago, sostiene que son las informaciones más que los comentarios las que hacen la opinión:

"Un periodista en posesión de hechos es un reformador más eficaz que un editorialista que se contenta con atronar en la cátedra, por más elocuente que sea". Bonnet (2015: 114).

Mientras que los comentarios periodísticos pueden tener orientaciones variadas en un mismo medio, las conclusiones a las que conducen las distintas noticias deben resultar coherentes entre sí en cada uno, lo que refuerza las constantes ideológicas del medio. En este sentido, un diario presenta una cosmovisión de lo real, producto de una enunciación llevada a cabo desde un posicionamiento (social, político, económico, ético, estético, entre otros), que Maingueneau (2009) atribuye a lo que designa como un 'metaenunciador': instancia de un nivel superior que está por encima de cada uno de los artículos que componen un diario o un noticiero televisivo o radial, que instala una identidad ethica con un grado importante de homogeneidad y regularidad en la publicación.

El periodista, para producir una noticia, debe trabajar el material informativo en bruto:

"Un testimonio no basta para hacer una información. [...] Esos materiales deberán ser trabajados, seleccionados, verificados, jerarquizados, contextualizados, iluminados y aclarados según las normas de la profesión” (Bonnet, 2015: 116).

De esta manera -y considerando el medio en el que se publica y la posición que desde ese lugar adopta el periodista frente a la información- el material pasa a ser noticia. Esta puede ser considerada, como un hipergénero, que se realiza en géneros diversos como la crónica política, policial o artística u otros géneros de información. La noticia se piensa como una unidad informativa por lo cual debe tener los datos necesarios para su comprensión:

"Un texto informativo debe explicarse en sí mismo. Ha de estar concebido de manera que el lector no necesite recordar los antecedentes para comprender la información que se le ofrece” (El País, 2014: 49).

Estos datos ineludibles ya están establecidos en los manuales, que lo recuerdan aunque utilicen mecanismos de atenuación: "El primer párrafo no debe contener 
necesariamente (aunque sí es conveniente) las clásicas respuestas a las preguntas qué, quién, cómo, dónde, cuándo y por qué”. Se piensa también la noticia como objetiva aunque la representación del hecho esté situada y sostenida por un sujeto: "La noticia es información sin interpretación. En ella se narran hechos que se supone son nuevos para el lector, mediante un relato ecuánime y sin implicación personal del periodista" (El País, 2014: 46). Otro manual (Libro de Estilo de El Mundo, 2014: 69) aclara: "El objetivo de la noticia es reflejar con la mayor exactitud posible la realidad a la que ha tenido acceso el periodista. Aunque la selección y colocación de elementos en una noticia implica necesariamente una elección subjetiva, no hay lugar para incluir opiniones o juicios de valor. Los rumores no son noticia”.

El desarrollo de nuevos medios y soportes ha multiplicado los modos de semiotización de la noticia (Millette, 2015). Además de las posibilidades hipertextuales que habilita, también se marcan los cambios en la composición y el estilo. Cuando la noticia se expone en lo que se considera el género crónica (en soporte papel) conserva los rasgos tradicionales: para orientar la interpretación deben explotarse tanto los elementos paratextuales como la orientación argumentativa del texto; y, así, poner en evidencia, desde su primer párrafo, la selección y la ubicación de los elementos considerados claves del evento mediante la construcción de un relato cuya orientación es reforzada en los titulares, las bajadas o las volantas. Pero lo que nos interesa señalar es que más allá de los rasgos genéricos y sus posibles transformaciones lo que sostiene a la noticia es el común dispositivo argumentativo que surge de un modo profesional de pensar y comunicar la información. El dispositivo consiste en un razonamiento entimemático en el que lo general convocado es la posición del diario y los datos informativos tienen la función de confirmarlo para llevar a una determinada conclusión sobre lo que se narra. El dispositivo puede desplegarse discursivamente de diferentes maneras aunque existan rutinas textuales ya establecidas.

Ejemplificamos a continuación con las noticias en la prensa gráfica sobre la revelación de los titulares de empresas offshore a través de los denominados 'Panamá Papers', que integró las tapas de distintos diarios de Argentina ${ }^{4}$ y del mundo el día 4 de abril de 2016. Fieles a las indicaciones de los distintos manuales, los titulares, bajadas, volantas y el primer párrafo presentan el foco de la notica que, como veremos, difiere en los distintos periódicos. El diario La Nación, de Argentina, propone el siguiente relato en su nota de tapa:

"Titular: Revuelo en el mundo: filtran datos de paraísos fiscales

Bajada: Involucran a políticos y funcionarios; Messi y Macri, entre los citados; para el Gobierno, no hubo delito

Primer párrafo: Por lo menos 140 políticos y funcionarios de todo el mundo, así como empresarios, miembros de la realeza, gente del espectáculo y el deporte quedaron ayer en la mira después de que la 
mayor filtración periodística en la historia revelara detalles sobre el desvío de fondos a paraísos fiscales.[...]

Cuarto párrafo: La filtración tuvo su eco en la Argentina. Del análisis de los documentos surgen, entre otros, los nombres de Mauricio Macri, que integró el directorio de una sociedad offshore en Bahamas y presidida por su padre, Franco Macri. La firma, Fleg Trading, operó hasta 2009 y según el Gobierno y expertos consultados por La Nación el Presidente no incurrió en delito."

Este medio pone el foco en la filtración de datos de paraísos fiscales que involucra entre otros a políticos, funcionarios, gente del espectáculo y del deporte de todo el mundo. Entre los involucrados, destaca en la bajada a un popular futbolista, Leonel Messi, y al actual presidente del país, Mauricio Macri. En el cuerpo de la nota, recién el cuarto párrafo se detiene en el presidente de la nación sobre quien informa que integró el directorio de una empresa offshore presidida por su padre que, aclara, 'operó hasta el 2009'. Atento a las controversias que este relato puede suscitar, la nota explicita la conclusión a la que busca llevar al lector, al final del párrafo cuarto, delegando la responsabilidad en las afirmaciones del gobierno y en la autoridad de los expertos: “... según el gobierno y expertos consultados por La Nación, el presidente no cometió ningún delito".

Otro matutino argentino de tirada nacional, Página/12, presenta la noticia también en su tapa pero asignándole al relato otra orientación argumentativa:

"Volanta: El presidente Mauricio Macri en el centro de un escándalo financiero internacional: forma parte del selecto grupo de cinco mandatarios en ejercicio, cuyos nombres figuran en la mayor filtración de la historia sobre sociedades off shore en 21 paraísos fiscales.

Titular: Argentina volvió al mundo

Bajada: El escándalo abarca a casi 70 mandatarios o ex mandatarios internacionales, como Putin y Cameron pero no directamente sino a través de allegados. También implica a otros políticos, artistas y deportistas como Messi y Platini.

Primer párrafo: (en el cuerpo del diario) Una filtración internacional de documentos sobre empresas que mueven dinero en paraísos fiscales involucró a Mauricio Macri, uno de los cinco mandatarios en funciones que figuran en la lista. El presidente argentino aparece como director en una empresa off shore en las Islas Bahamas, Fleg Trading Ltd, en la que también figura su padre, Franco Macri. Según especialistas en el área, este tipo de empresas suelen ser utilizadas para evasión impositiva. Macri no la incluyó en su declaración jurada cuando era jefe de gobierno porteño, a pesar de que figuró como director." 
Página/12, ante el caso Panamá Papers, pone el foco en el presidente de la nación. Remite, además, al momento en que Macri era jefe de gobierno en 2009 y se especifica que la empresa offshore no figuraba en su declaración jurada; aspectos todos que refuerzan la apreciación negativa sobre el presidente que se busca construir. El diario explicita el enunciado general que regula su razonamiento, atribuido a especialistas del área ('este tipo de empresas suelen ser utilizadas para la evasión impositiva'), el cual autoriza la conclusión implícita que se infiere del relato: el presidente cometió posiblemente un delito. Como puede observarse en la noticia de los dos medios, el evento narrado con orientaciones y focos diferentes dispara un razonamiento entimemático del que se derivan conclusiones opuestas en lo referido al presidente argentino.

El dispositivo argumentativo convierte lo particular en un relato verosímil, caracterizado como relevante en cuanto a su actualidad, y lo inscribe en un entimema, cuya conclusión generalmente no explícita deberá ser inferida por el lector. En el relato se representa lo ocurrido desde un sistema valorativo (propio de la posición del medio) sostenido sobre máximas, entendidas como enunciados generales de tipo ideológico (Angenot, 1989), que autorizan la inferencia de la conclusión. El caso analizado fue lo suficientemente polémico como para que las noticias explicitaran o bien la conclusión o bien el enunciado general para reforzar la interpretación.

\subsection{La ilustración de la norma o de su transgresión por el hecho probado, en el ámbito jurídico}

En el ámbito jurídico, lo particular que se rescata es aquello de lo cual se puede suministrar una prueba, que puede acompañar el escrito o ser obtenida fácilmente. Lo que, por ejemplo, en la demanda o la defensa, el cliente refiere es, como señala Carrió (1987: 20), "un borbollón de hechos" al que el abogado le tiene que otorgar un sentido para lo cual va a seleccionar, primero, aquellos que impliquen una relación de transgresión o de respeto a la norma (que expongan el alcance jurídico de la situación); y, luego, aquellos de los que pueda suministrar una prueba: lo particular no solo debe ser pertinente para el objeto del pedido y conducir al logro de una sentencia favorable a la posición del juez, sino también susceptible de ser acompañado por una prueba. En la sentencia funciona el mismo dispositivo argumentativo que va a permitir declarar la culpabilidad o inocencia de acusado.

En la extensa sentencia dictada contra la Junta Militar en la Argentina (9/12/1985), se enumeran los hechos y se suministran las pruebas:

"En los centros de cautiverio los secuestrados fueron interrogados en la casi totalidad de los casos bajo tormentos a través de métodos de tortura similares. 
[...] el tormento fue, en la enorme mayoría de los casos, la forma indiscriminadamente aplicada para interrogar a los secuestrados.

No existe constancia en autos de algún centro de cautiverio donde no se aplicaran medios de tortura y, en casi todos, la uniformidad de sistemas aparece manifiesta. Sólo pueden señalarse pequeñas variantes de tácticas o de modos, pero al pasaje de corriente eléctrica, los golpes y la asfixia, se repiten en casi la totalidad de los casos investigados, cualquiera sea la fuerza de la que dependía el centro o su ubicación geográfica.

Ello se prueba con los dichos de los testigos que han depuesto en la audiencia, como víctimas de tormentos, a saber: [...]."

Este tipo de despliegues discursivos constituye la parte central de la sentencia, pero presupone una selección, clasificación y evaluación de los hechos referidos por documentos y testimonios variados y de diferente procedencia (ya que se evalúan los aportados por las distintas partes). Son operaciones previas a la sentencia, que se exponen en materiales de diverso tipo, desde anotaciones a borradores de zonas del texto. En todos los casos lo que está presente para definir qué es lo que va a pasar al texto definitivo, es, primeramente, la relación hecho/prueba. En algunos casos puede no alcanzar el estatuto de prueba documentada y se señala su carácter de presunción que se afirma en pruebas indirectas:

“3) Habida cuenta de su naturaleza y características, no hay constancias documentales en autos de las órdenes secretas e ilegales que se han descripto en el apartado anterior.

Pese a ello, a juicio del Tribunal, su emisión resulta evidente a la luz de las múltiples e inequívocas presunciones que se señalan a continuación:

a) Los propios comandantes alegaron haber tenido el control efectivo de sus fuerzas y negaron la existencia de grupos militares que actuaran con independencia de la voluntad del comando, circunstancias ambas que no fueron desvirtuadas en la causa.

b) La totalidad de los jefes y oficiales que han declarado en la audiencia o en actuaciones agregadas afirmaron que la lucha antisubversiva se ajustó estrictamente a las órdenes de sus comandantes superiores. [...]

Así lo afirmaron en la audiencia los brigadieres [...]; los contralmirantes $[\ldots]$; el Almirante $[\ldots]$; los generales $[\ldots]$; los coroneles $[\ldots]$; y en las actuaciones $[\ldots]$.

c) El sistema operativo puesto en práctica -captura, interrogatorios con tormentos, clandestinidad e ilegitimidad de la privación de libertad y en muchos casos eliminación de las víctimas- fue sustancialmente idéntico en todo el territorio de la Nación y prolongado en el tiempo.

Encontrándose probado que los hechos fueron cometidos por miembros de las fuerzas armadas y de seguridad, organizadas vertical y 
disciplinadamente, resulta descartable la hipótesis de que pudieron haber ocurrido sin órdenes expresas de los superiores."

Por otra parte, los hechos probados deben poder ser referidos a un marco jurídico, de allí la importancia de la relación entre ellos y la norma, la jurisprudencia o la doctrina: "Los hechos se relacionan inmediatamente con la prueba, así como el derecho se relaciona con la inclusión del hecho en la norma jurídica" (Falcón, 1997: 44). Este doble juego es lo que va a sostener la conclusión:

"Ha quedado acreditado que a gran parte de los cautivos se los sometió a distintos tipos de vejaciones físicas con el propósito de obtener información, en algunos casos, o de quebrar su fuerza de voluntad, en otros, cuando ya no había datos que obtener.

[...] Tales hechos constituyen el delito de imposición de tormentos, previsto en el artículo 144ter, primer párrafo, del Código Penal, según texto introducido por la ley 14.616, vigente a la época de comisión de los hechos, por resultar más benigno que el actual, que obedece a la ley 23.077 (artículo 2 ibídem).

La exigencia de que los sufrimientos sean causados con un propósito determinado - obtener información o quebrar la voluntad - (v. Ricardo C. Nuñez, op. cit., T.V: 57; Sebastián Soler, op.cit., ed. 1970, T.IV: 52; Carlos Fontán Balestra, op. cit., ed.1980, T.V: 318) se ve satisfecha, pues ellos fueron llevados a cabo con las finalidades señaladas más arriba, a sabiendas de lo que se estaba haciendo."

Ingresar el hecho al dominio jurídico exige interrogar el universo normativo y modelar el hecho para que se adecue a él. En algunas situaciones, la adecuación a la norma puede plantear dudas, lo cual obliga a interrogarla, a evaluarla en relación con otras o a señalar los vacíos legales. En esta situación, el dispositivo argumentativo dominante será el del caso, en un uso similar al que observamos en el campo psicoanalítico, con un movimiento ilustrativo (en el que el reconocimiento de los hechos probados y su relación con la normas son insoslayables) y otro que devela la inadecuación de algunos aspectos y la necesidad de apelar a otra normativa o formular un enunciado susceptible de ser generalizado que permita arribar a una conclusión. En el caso jurídico, a diferencia del psicoanalítico lo conjetural se anula para dar lugar a la toma de decisiones que van a sentar jurisprudencia.

\section{COMENTARIOS DE CIERRE}

A lo largo del artículo hemos querido mostrar el interés de abordar los modos de pensar la relación entre lo particular (los hechos que se seleccionan) y lo general (la teoría, la doctrina, el posicionamiento ideológico, las normas) para elaborar una respuesta a los desafíos propios de cuatro profesiones en las que la producción discursiva ocupa un lugar privilegiado: psicoanalistas, sacerdotes, periodistas y 
abogados. Hemos llamado a los tipos de razonamiento considerados 'dispositivos argumentativos' no solo porque tienden a una conclusión -neta o vacilante, explícita o implícita- sino también porque funcionan como matrices intelectuales del despliegue discursivo. Ellos sostienen los géneros centrales de cada práctica (relato clínico, homilía, noticia y sentencia) pero también están presentes en muchos otros: los que, en Psicoanálisis, exponen casos; en la predicación, actualizan el mensaje; en el periodismo, refieren las noticias; o, en el ámbito jurídico, fundamentan el respeto o la transgresión a la norma. Incluso inciden fuertemente en otros géneros no propios de la profesión pero en los que los expertos pueden intervenir, como lo hemos ilustrado con la nota periodística de un sacerdote.

Consideramos que la reflexión sobre los dispositivos argumentativos complementa en la formación profesional las actividades centradas en la pedagogía de géneros (Venegas, Núñez, Zamora \& Santana, 2015), atenta a los rasgos retórico-discursivos propios de cada uno. La elaboración de respuestas eficaces depende no solo del desarrollo de la habilidad de redactar escritos sino también del dominio de los modos de razonar que cada profesión ha ido consolidando. Si bien en las respectivas carreras, los estudiantes van aprendiendo prácticamente cómo se reflexiona en los ámbitos propios, el vínculo entre operaciones intelectuales y semiotización no se aborda explícitamente. Los talleres de escritura pueden resultar ámbitos adecuados para tal tarea, si nos proponemos formar profesionales que puedan realizar intervenciones innovadoras respecto de los problemas diversos que cada esfera de actividad plantea y no meros usuarios de programas de generación automática de textos. 


\section{REFERENCIAS BIBLIOGRÁFICAS}

Angenot, M. (1989). La parole pamphlétaire. París: Payot.

Arnoux, E. (2009). Funciones de la escritura en el capítulo de tesis y en la defensa oral de la tesis. En E. Arnoux (Dir.), Escritura y producción de conocimiento en posgrado (pp. 143-168). Buenos Aires: Santiago Arcos.

Arnoux, E. (2012). Potencialidades y limitaciones de los dispositivos argumentativos que articulan materiales clínicos y reflexión teórica en los escritos del campo psicoanalítico. Cogency. Journal of Reasoning and Argumentation, 4(2), 47-75.

Arnoux, E. (2015a). Los manuales de estilo periodísticos para las versiones on line: Las representaciones del lector y su incidencia en la regulación de discursos y prácticas. Circula. Revista de Ideologías Lingüísticas, Vol. 2, 138-160, Éditions de l'Université de Sherbrooke (ÉDUS).

Arnoux, E. (2015b). Escritura y predicación: La homilía como género de la celebración litúrgica, Traslaciones, 2(4), 67-93.

Arnoux, E., Blanco, I. \& di Stefano, M. (1999). Las representaciones de la lengua y de la prensa en los manuales de estilo periodísticos argentinos. En E. Arnoux \& R. Bein (Comps.), Prácticas y representaciones del lenguaje (pp.175-190). Buenos Aires: Eudeba.

Arnoux, E., di Stefano, M. \& Pereira, C. (2010). Materiales clínicos y supervisión: Escritos del campo psicoanalítico. En G. Parodi (Ed.), Alfabetización académica y profesional: Leer y escribir desde las disciplinas (pp.185-214). Santiago de Chile: Ariel.

Auroux, S. (1994). La révolution technologique de la grammatisation. Lieja: Mardaga.

Bazerman, Ch. (2009). Gêneros textuais, tipificação e interação. San Pablo: Cortez Editora.

Bazerman, Ch. (2015). Teoria da ação letrada (Vol. 1) y Retórica da ação letrada (Vol. 2). San Pablo: Parábola Editorial.

Belluscio, A. (2006). Técnica jurídica para la redacción de escritos y sentencias. Reglas gramaticales. Buenos Aires: Sup. Esp. y Sent.

Bonnet, F. (2015). Mediapart, un journal au coeur de la révolution numérique. En R. Maltais \& P. Cayouette (Dirs.), Les journalistes. Pour la survie du journalisme (pp. 109-124). Quebec: Ediciones Québec Amérique.

Borel, M-J. (2000). Métaphores et analogies dans le discours des sciences de l'homme et de la société. Revue Européenne des Sciences Sociales, XXXVIII, 117. 
Borja Albi, A. (2007). Los géneros jurídicos. En E. Alcaraz (Ed.), Las lenguas profesionales y académicas (pp. 140-153). Barcelona: Ariel.

Burdiles, G. (2015). La organización retórica de la macromovida Relato del Caso en el género Caso Clínico en español: Convenciones y desacuerdos en nueve especialidades médicas. En G. Parodi \& G. Burdiles (Eds.), Leer y escribir en contextos académicos y profesionales (pp.187-220). Santiago de Chile: Ariel.

Carrió, G. (1987). Cómo estudiar y cómo argumentar un caso. Buenos Aires: Abeledo-Perrot.

Falcón, E. M. (1997). Cómo hacer una demanda. Buenos Aires: Abeledo-Perrot.

Fernández, V. M. (2009). Cómo interpretar y cómo comunicar la palabra de Dios. Métodos y recursos prácticos. Buenos Aires: San Pablo.

Francisco, Santo Padre (2013). Exhortación apostólica EVANGELII GAUDIUM del Santo Padre FRANCISCO a los obispos, a los presbiteros y diáconos, a las personas consagradas y a los fieles laicos sobre el anuncio de Evangelio en el mundo actual. Roma: Tipografía Vaticana.

Gardes Tamine, J. (2011). Pour une nouvelle théorie des figures. París: Presses Universitaires de France.

Garzoli, E. (1996). La adicción de transferencia. Acerca del análisis de una paciente alcohólica. Psicoanálisis APdeBA, Vol XVIII N², 237-258 [en línea]. Disponible en: http://biblioapdeba.no-ip.org/pgmedia/EDocs/1996revista2-Garzoli

Gitaroff, G. (2010). Escribir sobre la clínica. En G. Gitaroff (Ed.), Claves para escribir sobre Psicoanálisis (pp. 101-134). Buenos Aires: Letra Viva.

Jolles, A. (1972 [1930]). Formes simples. París: Seuil.

Maingueneau, D. (2009). Análisis de textos de comunicación. Buenos Aires: Nueva Visión.

Miéville, D. (1983). Analogie et exemple. En M.-J. Borel, J.-B. Grize \& D. Miéville (Eds.), Essai de logique naturelle (pp. 147-214). Berna: Peter Lang.

Millette, L. (2015). L'ADN du journalisme: En voie de mutation ou non? En R. Maltais \& P. Cayouette (Dirs.), Les journalistes. Pour la survie du journalisme (pp. 89-100). Québec: Ediciones Québec Amérique.

Passeron, J-C. \& Revel, J. (Dirs.) (2005). Penser par cas. París: Enquête.

Paveau, M-A. (2006). Les prédiscours. Sens, mémoire, cognition. París: Presses Sorbonne Nouvelle. 
Pereira, M. C. (2011). Interacción argumentativa y práctica profesional: La presentación de casos clínicos en revistas de especialidad. Actas XVI Congrego Internacional Alfal (pp. 4067-4076) [en línea]. Disponible en: http://alfal2011.mundoalfal.org/\#/pdf/459alfal.pdf

Perelman, Ch. \& Olbrechts-Tyteca, L. (1970). Traité de l'argumentation. La nouvelle rhétorique. Bruselas: Editions de l’Université de Bruxelles.

Roy, J-H. (2015). Le grand dérangement numérique: Plaidoyer por un journalisme hacker. En R. Maltais \& P. Cayouette (Dirs.), Les journalistes. Pour la survie du journalisme (pp. 125-142). Québec: Ediciones Québec Amérique.

Venegas, R., Núñez, M. T., Zamora, S. \& Santana, A. (2015). Escribir desde la pedagogía del género. Guías para escribir el trabajo final de grado en licenciatura. Valparaíso: EUV.

\section{NOTAS}

${ }^{1}$ En esta ocasión no vamos a considerar las articulaciones intersemióticas, sino que vamos a centrarnos en el aspecto verbal escrito, ya que la reflexión busca aportar a una pedagogía de la escritura.

${ }^{2}$ La traducción es nuestra pero el resalte es de la autora.

${ }^{3}$ Para el análisis del género: Arnoux, di Stefano y Pereira (2010).

4 Pueden consultarse on line las noticias, que analizamos, de ese día sobre el tema en:

http://servicios.lanacion.com.ar/archivo y en

http://www.pagina12.com.ar/usuarios/anteriores.php 